\title{
INVENTÁRIO SIMPLIFICADO DE QUALIDADE DE VIDA NA EPILEPSIA INFANTIL: PRIMEIROS RESULTADOS
}

\author{
PAULA TEIXEIRA FERNANDES*, ELISABETE ABIB PEDROSO DE SOUZA**
}

\begin{abstract}
RESUMO - Objetivo: Verificar como os pais percebem a qualidade de vida dos filhos epilépticos e se crenças relativas à epilepsia controlam a relação pais e filhos. Método: Foram aplicados 21 protocolos do "Inventário Simplificado de Qualidade de Vida na Epilepsia Infantil" em pais de crianças com 6 a 14 anos portadoras de epilepsia benigna da infância. Resultados: Observou-se que $86 \%$ dos pais relataram crenças relativas à epilepsia. A maioria dos pais avaliou a qualidade de vida dos filhos como muito boa, mas relataram dificuldades no lidar com eles. Aparecem comportamentos de superproteção (62\%) e sentimentos de preocupação, medo e insegurança (90\%). As crianças foram avaliadas como irritadas (52\%), dependentes (38\%), agitadas e inquietas (38\%). No que diz respeito à escola, $33 \%$ das crianças freqüentam escolas especiais e apresentam dificuldades escolares e de relacionamento. Conclusão: Crenças e desinformação parecem controlar o comportamento dos pais na maneira de lidar com seus filhos epilépticos, gerando comportamentos inadequados, apesar disto, percebem a qualidade de vida dos mesmos como muito boa.
\end{abstract}

PALAVRAS-CHAVES: epilepsia infantil, qualidade de vida, relação pais-filhos.

\section{Simplified inventory of quality of life in childhood epilepsy: initial results}

ABSTRACT - Purpose: to check how parents realize the quality of life of their epileptic children and if the relationship is controlled by their beliefs about epilepsy. Method: it has been applied 21 protocols of "Simplified inventory of quality of life in childhood epilepsy" to parents of children aged between 6-14 years old with benign childhood epilepsy. Results: it was observed that $86 \%$ of parents reported beliefs about epilepsy. The majority of parents evaluated their children's quality of life as very good, but reported difficulties to deal with them. Behaviors of overprotection (62\%) and feelings of worry, fear and insecurity (90\%) were observed. The children were evaluated as irritated (52\%), dependents (38\%), overwroughts and inquiets (38\%). About school, 33\% are in special schools and have difficulties of academic and relationship. Conclusion: it was verified a lot of beliefs and acknowledgement seem to control parent's behavior on the way of dealing with epileptic children producing inadequate behaviors, in spite of realizing the quality of life of their children as been very good.

KEY WORDS: childhood epilepsy, quality of life, parents-child relationship.

Qualidade de vida tem sido diferentemente conceituada como a realização de um plano de vida, como satisfação de necessidades, mas será entendida neste estudo como senso de bem estar ${ }^{1}$. Há inúmeros fatores que podem ter influência na qualidade de vida das crianças epilépticas, não só os fatores relacionados diretamente à epilepsia (tipo e frequência das crises, etiologia, tratamento, medicamentos, cuidados médicos), como também os fatores psico-sócio-culturais (comportamentos, relacionamento familiar e escolar, crenças, emoções) ${ }^{2}$.

A qualidade da interação entre pais e filhos é responsável pelos problemas de comportamento e pelas competências da criança em casa e na escola. Crianças com epilepsia têm alta prevalência de comportamento alterado ${ }^{3}$ e dificuldades de aprendizagem ${ }^{4}$ que aparecem relacionadas com

Departamento de Neurologia da Faculdade de Ciências Médicas (FCM) da Universidade Estadual de Campinas (UNICAMP): *Psicóloga, mestranda em Neurociências; **Psicóloga, Professor Assistente Doutor. Aceite: 19-outubro-1998.

Paula Teixeira Fernandes - Departamento de Neurologia/FCM/UNICAMP - Caixa Postal 6111 - 13083-970 Campinas SP - Brasil. E-mail: famfern@correionet.com.br 
dificuldades interacionais entre pais e filhos. O desenvolvimento social e emocional prejudicados, consequentemente, alteram a qualidade de vida da criança, sua adaptação ao tratamento e seu ajustamento psicossocial ${ }^{5}$. Muitos estudos falam de evidente relação entre a doença e as variáveis do contexto familiar, envolvidas num processo de ajustamento ${ }^{6}$. Estudos de Lothman e Pianta ${ }^{7}$ e Austin e col. ${ }^{8}$ observaram a relação existente entre o ajustamento das crianças epilépticas com as atitudes dos pais. Relações familiares, principalmente entre mãe e filho têm importante significado na autoestima, interação social, dependência e habilidades acadêmicas ${ }^{6}$. Uma outra variável importante apontada por Souza e Guerreiro ${ }^{1}$ diz respeito aos lapsos nas explicações sobre a epilepsia, pois reforçam os medos, trazem insegurança e podem influenciar negativamente o ajustamento comportamental e psicossocial da criança, pois alteram a forma de lidar com o filho epiléptico. Muitas vezes, problemas interacionais podem ser prejudicados pela falta de informação sobre a doença, gerando crenças e sentimentos de insegurança, preocupação, medo e superproteção dos pais no lidar com seus filhos epilépticos ${ }^{1}$.

O objetivo deste estudo foi verificar, junto a pais de crianças epilépticas, como percebem a qualidade de vida dos seus filhos e se crenças relativas à epilepsia controlam a relação pais e filhos.

\section{MÉTODO}

\section{Sujeitos}

Os pais que acompanhavam seus filhos nas consultas do ambulatório de epilepsia infantil do HC/UNICAMP foram encaminhados para o setor de psicologia para participarem voluntariamente de grupos de apoio. Estes grupos, supervisionados por uma psicóloga, têm em média uma hora de duração, onde são discutidos aspectos psicológicos e informações relativas à epilepsia.

Os primeiros 21 pais que preencheram os critérios de seleção, e concordaram em participar da pesquisa, foram submetidos à aplicação do inventário. Foram selecionados pais cujas crianças tivessem de 6 a 14 anos e fossem portadoras de epilepsia benigna da infância. As crianças tinham em média 10 anos e 4 meses (idades de 6 a 14 anos) e o tempo da doença variava de 1 a 10 anos (média 4 anos e 9 meses).

\section{Instrumento}

O "Inventário Simplificado de Qualidade de Vida na Epilepsia" avalia dados pessoais da criança (idade, escolaridade, pais), variáveis da doença (tipo da epilepsia, tempo da doença, medicação, controle e frequência das crises), variáveis culturais (crenças irracionais), variáveis psicológicas e sociais (comportamento da criança em casa e na escola, comportamento dos pais em relação aos filhos, relacionamento social e escolar da criança) e a percepção da qualidade de vida das crianças com epilepsia (apresentada numa escala de 1 a 10$)^{9}$.

Este inventário foi elaborado a partir do "Inventário de Qualidade de Vida" de Guerreiro e col. ${ }^{4}$ e é baseado no relato dos pais de crianças portadoras de epilepsia, sobre aspectos importantes que afetam a vida de seus filhos, permitindo a identificação do impacto da doença no comportamento da criança e na dinâmica familiar.

\section{RESULTADOS}

No que se refere aos aspectos culturais, pôde-se perceber que $86 \%$ dos pais apresentam respostas que identificam a respeito da epilepsia. Aparecem comportamentos supersticiosos como alternativa de tratamento em $24 \%$ dos pais. As crenças que mais foram relatadas dizem respeito à possibilidade da criança engolir a língua durante a crise (33\%) e à possibilidade das crises ocorrerem porque o filho possui algum verme (29\%).

Em relação aos aspectos pessoais, os dados revelam que $76 \%$ dos pais consideram seus filhos como pessoas normais, mas apresentam medo da ocorrência de nova crise (71\%), medo de contrariar o filho (43\%) e comportamentos de superproteção (62\%).

No que diz respeito à qualidade de vida, a maioria dos pais avaliou-a como muito boa ( $48 \%$ deram nota dez, $19 \%$ deram nota nove, $19 \%$ deram nota oito), mas relataram dificuldades no lidar com seus filhos. Aparecem sentimentos de preocupação, medo e insegurança em $90 \%$ dos pais.

Em relação aos aspectos pessoais, as crianças foram avaliadas como irritadas (52\%), dependentes (38\%), agitadas e inquietas (38\%), agressivas (19\%) e com poucos amigos (19\%). (Obs: houve pais que associaram mais de um comportamento). 
No que se refere a escola, 33\% das crianças frequentam escolas especiais e foi relatado que elas apresentam dificuldades acadêmicas e de relacionamento com os colegas. Entre as crianças com escolaridade adequada, houve queixas de memória em 19\% dos casos.

\section{DISCUSSÃO}

A epilepsia é doença crônica comum na infância e é considerada uma experiência frustrante para a família, pois influencia o comportamento da criança e o ajustamento familiar ${ }^{10}$. A frequência de comportamentos alterados na criança influencia a qualidade de vida dela.

Alguns autores ${ }^{11,12}$ sugerem que o uso de drogas antiepilépticas pode comprometer o dia-adia das crianças, porque seus efeitos afetam o comportamento e as funções cognitivas, podendo causar irritabilidade, problemas de aprendizagem, hiperatividade, ansiedade e desordens de conduta. Entretanto, a maioria dos estudos concorda quanto a importância de variáveis psicossociais como determinantes dos problemas de comportamento ${ }^{6,13,14}$.

Neste estudo, foi possível a identificação de comportamentos alterados a nível acadêmico e de relacionamento. A maioria das crianças apresenta escolaridade adequada (apenas $33 \%$ frequentam escolas especiais e apenas $19 \%$ apresentam queixas de memória). Este dado mostra que as alterações apresentadas não estão relacionadas com algum déficit intelectual, mas com a possibilidade da presença de estigma relacionado à doença e consequentes reações inadequadas dos pais e professores. O não saber lidar com a epilepsia pode gerar baixas expectativas nos pais e professores e uma baixa auto-estima nas crianças portadoras de epilepsia ${ }^{1}$.

No que diz respeito ao comportamento das crianças no relacionamento familiar e social, elas foram avaliadas como irritadas, dependentes, inquietas e agitadas, agressivas. Os pais relataram sentimentos de preocupação, medo, insegurança e ansiedade que parecem ser gerados pelo número excessivo de crenças $(86 \%)$ relacionadas à epilepsia. A presença de superproteção e comportamentos inadequados reforçam a hipótese de inabilidades dos pais em lidar com suas crianças, decorrentes de sentimentos e crenças irracionais. Esses dados coincidem com os de Guerreiro e col. ${ }^{2}$ que identificaram significante presença de alteração de comportamento nas crianças após o início da doença, contingente às atitudes dos pais. Keith ${ }^{15}$ também evidenciou sentimentos de inadequação, raiva, confusão relacionados a atitudes de superproteção nos pais.

Algumas idéias controlam os comportamentos dos pais. Idéias provenientes de uma infinidade de fontes (vizinhos, parentes, amigos) cuja validade raramente é checada com médicos e profissionais envolvidos no tratamento. No presente estudo muitos pais apresentaram crenças irracionais relativas ao medo da criança engolir a língua. De acordo com Ford e col. ${ }^{14}$, essa idéia é bastante comum e os pais costumam colocar canetas, dedos, réguas na boca da criança. Por isso, quando expõem para os profissionais suas dúvidas e ansiedades, podem ser instruídos em como agir em situações de crise, evitando assim estresse e tensão familiar.

Muitas vezes, alguns conceitos errados não são expostos pelos pais ou não são identificados pelos médicos. O uso de um instrumento de avaliação da qualidade de vida pode permitir essa identificação e com isso, a possibilidade de uma orientação adequada que saliente a importância do tratamento da epilepsia tanto a nível médico, como a nível psicológico.

Thompson e Upton ${ }^{16}$, evidenciaram que os pais, geralmente, são um pouco confusos no que diz respeito à epilepsia; para eles essa desordem, muitas vezes, tem má conotação, pois é associada com doença ou retardo mental. Existe um alto nível de ansiedade que é alimentado por mitos e preconceitos sobre a doença e pelo medo da epilepsia vir acompanhada de sérios problemas de personalidade. Informações que são totalmente errôneas e acarretam sérios danos às pessoas. A presença de uma crença irracional é suficiente para gerar ansiedade e comportamentos inadequados ${ }^{17}$. Uma reação comum dos pais é a superproteção, que pode ser mais prejudicial do que as próprias crises. Muitas vezes, os pais precisam de permissões médicas e de encorajamento para impor limites. Por medo das consequências, precisam de estímulo para tratar as crianças com normalidade ${ }^{14}$. 
Quando o médico, além de avaliar os sinais da doença, abre espaço para o paciente e/ou familiares colocarem suas dificuldades a respeito de estar doente ou de ter alguém doente em casa, amplia a perspectiva de atendimento integral ao paciente e sua família, prevenindo uma relação inadequada entres os mesmos, através de informações corretas.

Quanto à percepção de qualidade de vida das crianças com epilepsia, a maioria dos pais definiu esta variável como muito boa. Provavelmente, estes pais estavam se baseando no fato das crises estarem sob controle e de suas crianças estarem levando uma vida normal, frequentando escolas, e não a relacionaram com as dificuldades de interação e problemas de comportamento por eles relatados.

Entrevistas com os pais, muitas vezes, permitem conhecer não só medidas de qualidade de vida, como também aspectos importantes que afetam as relações e o comportamento das crianças com epilepsia. Seria importante confirmar os dados obtidos através do relato dos pais com a própria criança, obtendo novas informações que complementam informações a respeito do real impacto da doença na vida da criança e sua percepção das mudanças familiares.

A investigação de variáveis familiares em relação ao controle de comportamentos e ajustamento à doença é muito importante. A identificação destas permite a prevenção de comportamentos inadequados e, consequentemente, a melhora na qualidade de vida das crianças epilépticas e suas famílias. A desmistificação de crenças e preconceitos, através de informações corretas sobre a doença e seu tratamento, diminui a ansiedade da família, garante mais cuidados eficazes no atendimento à criança com epilepsia e promove melhor ajustamento dela ${ }^{13,16}$.

Algumas vezes, a criança sente-se estigmatizada por causa da reação dos pais ou de outras pessoas próximas. Quando se identifica precocemente estas variáveis, é possível atuar de modo mais positivo na dinâmica familiar, controlando o ajustamento da criança e da família à epilepsia.

O "Inventário Simplificado de Qualidade de Vida na Epilepsia" é de fácil aplicação em uso clínico, com linguagem clara para o entendimento dos sujeitos e permite a identificação de importantes aspectos que atuam no cotidiano da criança com epilepsia e sua família. A padronização do instrumento será feita posteriormente, à medida que novos casos de epilepsia forem sendo avaliados.

\section{REFERÊNCIAS}

1. Souza EAP, Guerreiro MM. Qualidade de vida e epilepsia. In Guerreiro CAM, Guerreiro MM (eds). Epilepsia. 2.Ed. São Paulo: Lemos Editorial 1996;18:191-199.

2. Guerreiro MM, Silva EA, Scotoni AE, Souza EAP. Qualidade de vida em epilepsia na infância. JLBE 1994;7:21-26.

3. Austin JK. Comparison of child adaptation to epilepsy and asthma. J Child Adolesc Psychiatr Ment Health Nurs 1989;2:139-144

4. Seidenberg M, Beck N, Geisser M. Academic achievement of children with epilepsy. Epilepsia 1986;27:753-759.

5. Hoare P, Kerley S. Psychosocial adjustment of children with chronic epilepsy and their families. Dev Med Child Neurol 1991;33:201-215

6. Lothman DJ, Pianta RC. Role of child-mother interaction in predicting competence of children with epilepsy. Epilepsia 1993;34:658-669.

7. Lothman DJ, Pianta RC. Predicting behavior problems in children with epilepsy: child factors, disease factors, family stress and chid-mother interaction. Child Dev 1994;65:1415-1428.

8. Austin JK, McBride AB, Davis HW. Parental attitude and adjustment in childhood epilepsy. Nurse Res 1984;33:92-96.

9. Souza EAP. Qualidade de vida na epilepsia infantil. Arq Neuropsiquiatr 1998,57:34-39.

10. Souza EAP, Nista CR, Scotoni AE, Guerreiro MM. Sentimentos e reações de pais de crianças epilépticas. Arq Neuropsiquiatr 1998;56:39-44.

11. Austin JK, Smith MS, Risinger MW, McNellis AM. Childhood epilepsy and asthma: comparison of quality of life. Epilepsia 1994;35:608-615.

12. Cull CA, Trimble MR, Wilson J. Changes in antiepilepsy drug regimen and behavior in children with epilepsy. J Epilepsy 1992;5:1-9.

13. Hanai T. Quality of life in children with epilepsy. Epilepsia 1996;37(Suppl 3):28-32.

14. Ford CA, Gibson P, Dreifuss FE - Psychosocial considerations in childhood epilepsy. In Dreifuss (ed). Pediatric epiteptology classification and management of seizures in the child. Massachussets: John Wright \& Sons, 1984;18:277-295.

15. Keith RM. The feelings and behaviour of parents of handicapped children. Dev Med Child Neurol 1978;4:524-527.

16. Thompson PJ, Upton D. Quality of life in family members of persons with epilepsy. In Trimble MR and Dodson WE (eds). Epilepsy and quality of life. New York: Raven Press, 1994:19-30.

17. Beck AT, Rush AJ, Shaw BF, Emery G. Cognitive therapy of depression. Zahar Eds, 1982. 